\title{
SMALL-ANGLE NEUTRON SCATTERING STUDIES OF THE TEMPLATE-MEDIATED CRYSTALLIZATION OF ZSM-5 TYPE ZEOLITE'
}

\author{
L. E. Iton 1*, F. Trouw2 2 , T. O. Brun 1 , and J. E. Epperson 1
}

Materials Science Division and Pulsed Neutron Source Division

Argonne National Laboratory, Argonne, Illinois 60439, U, S, A.

J. W. White ${ }^{3 *}$ and S. J. Henderson
Research School of Chemistry

The Australian National University, Canberra, Australia:-

\section{March 1988}

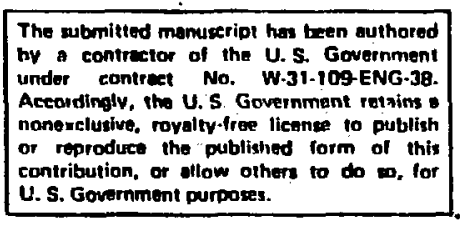

* Authors to whom correspondence should be addressed.

1 Materials Science Division, Argonne National Laboratory

2 Pulsed Neutron Source Division, Argonne National Laboratory

3 Argonne National Laboratory Fellow

$\uparrow$ Work supported in part by the U.S. Department of Energy, BES-Materials Science, under
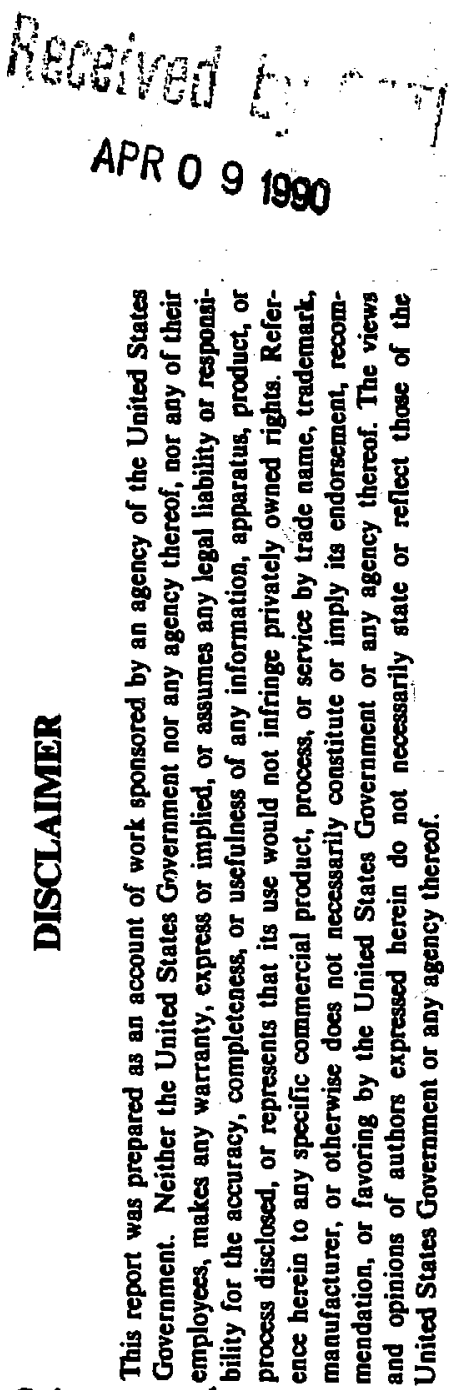
contract No. W-31-109-ENG-38; performed in part at the neutron beam facilities of the Institut Laue - Langevin and the Intense Pulsed Neutron source at Argonne National Laboratory. 


\title{
SMALL-ANGLE NEUTRON SCATTERING STUDIES OF THE TEMPLATE-MEDIATED CRYSTALLIZATION OF ZSM-5 TYPE ZEOLITE ${ }^{\dagger}$
}

\author{
L. E. Iton $1 *$, F. Trouw 2 , T. O. Brun 1 , and J. E. Epperson 1 \\ Materials Science Division and Pulsed Neutron Source Division \\ Argonne National Laboratory, Argonne, Illinois 60439, U. S. A. \\ J. W. White ${ }^{3 *}$ and S. J. Henderson \\ Research School of Chemistry \\ The Australian National University, Canberra, Australia.
}

\section{SUMMARY}

Small-angle neutron scattering is a useful new approach to the study of zeolite crystallization from aluminosilicate gels and the action of template molecules. It has been applied to gels for synthesis of zeolite ZSM-5 using tetrapropylammonium ions as templates where the scattering length densities of the gel particles and their texture were determined using contrast variation methods. Gels formulated from soluble silicate incorporate template molecules promptly into an amorphous "embryonic" structure and crystallization ensues via a solid hydrogel transformation mechanism. Gels formulated from colloidal silica show different scattering behavior, and a liquid phase transport mechanism is inferred.

* Authors to whom correspondence should be addressed.

1 Materials Science Division, Argonne National Laboratory

2 Pulsed Neutron Source Division, Argonne National Laboratory

3 Argonne National Laboratory Fellow

$\dagger$ Work supported in part by the U. S. Department of Energy, BES-Materials Science, under contract No. W-31-109-ENG-38; performed in part at the neutron beam facilities of the Institut Laue - Langevin and the Intense Pulsed Neutron source at Argonne National Laboratory. 
ZSM-5 zeolite is an important shape-selective zeolite which is best synthesized by hydrothermal crystallization of an aluminosilicate gel in the presence of tetrapropylammonium (TPA) cations as template molecules. The (nominally) aluminium-free version, silicalite, can also be synthesized. The exact role of template molecules in zeolite crystallization is as yet unclear, and details of gel structure and crystal nucleation mechanisms are not well understood. This presents an important challenge since it is recognized that an understanding of the details of crystallization mechanisms and gel chemistry is a prerequisite to being able to synthesize, by design, zeolites of prescribed crystal structure. Several physical techniques have recently been employed in the quest to characterize species in zeolite synthesis gels, notably magic-anglespinning nuclear magnetic resonance (MAS-NMR) ${ }^{1-3}$ spectroscopy and laser Raman spectroscopy 4,5 . The emphasis has been on the attempt to identify silicate molecular species in the gel which presage the development of particular framework structures, and on systematizing the chemical variables which control the various syntheses. We will demonstrate in this paper how the technique of small-angle neutron scattering (SANS) can provide some insights into the nature of gels and crystallite nucleation which are not readily extracted via spectroscopic methods.

The neutron scattering lingth is a nuclear property of individual atoms, so that molar composition and physical density of a substance uniquely determine its scattering length density, $P_{m}$. The intensity $(\mathrm{I}(\mathrm{Q}))$ of the small-angle angle scattering from a gel depends on the difference in scattering length density $\left(\rho_{m}-\rho_{S}\right)$ between the solid $\left(\rho_{m}\right)$ and nuid $\left(\rho_{S}\right)$ phases, as given 6 by :

$$
I(Q)=K\left(\rho_{m}-\rho_{S}\right)^{2} \cdot S(Q)
$$

where $Q$, the scattered neutron momentum transfer, is given by $Q=(4 \pi \sin \theta) / \lambda ; 2 \theta$ is the scattering angle, and $S(Q)$ is the scattering function 6 from the atomic particle distribution in the sample. At high contrast, this is the atomic distribution in the gel. By exploiting isotopic labelling of the fluid and the solid with hydrogen and deuterium, which have a very large 
difference in their scattering lengths, the scattering intensity as a function of fluid scattering length density can be measured. At the contrast match condition, the scattering length densities of the fluid and solid components match, $\rho_{m}-\rho_{s}=0$, and the scattering intensity due to the solid particles vanishes. The scattering length density of the solid particles is thus determined. Computed scattering length densities for crystalline silicalite containing h-TPA and d-TPA are listed in Table 1, along with the values for dense amorphous silica and for silicalite with no TPA. It is apparent that one can arrange to contrast match a particular expected component of a formed gel and thereby observe selectively the scattering due to a component which has a significantly different scattering length density.

In this paper, the above methods are applied to examine two different aluminosilicate (or silicate) synthesis gels for ZSM-5 in the presence and absence of TPA. Contrast variation over a wide range has been achieved by use of both hydrogenated (h-TPA) and deuterated (d-TPA) templates and variations in the $\mathrm{D}_{2} \mathrm{O}: \mathrm{H}_{2} \mathrm{O}$ ratio of the fluid media. Complementary measurements were made with X-ray powder diffraction (XRD) and Fourier transform infrared (FTIR) spectroscopy to check the degree of crystallinity of the gels. Our aim is to distinguish between the two extreme mechanisms have been proposed for crystallization of zeolite synthesis gels 1 . The nature of the siliceous component used in forming the gel is of paramount importance in determining which mechanism prevails in a synthesis. In the liquid phase ion transport mechanism, the zeolite crystallites are nucleated from template cations and (alumino)silicate anions redissolved in the liquid phase by slow depolymerization of the gel particles. This mechanism should prevail when the gel is formed using colloidal silica as an ingredient. In the solid hydrogel reconstruction mechanism, the crystallites arise by a direct crystallization of the gel, presumably via surface nucleation. Thus the hydrogel particles initially formed could directly incorporate the template molecules, and this mechanism should prevail when the gel is formed from soluble monomeric (or low oligomeric) silicate anions. 
The SANS patterns from unheated gels formed with a sodium silicate solution as reagent were measured on the small-angle diffractometer D17 and the long-wavelength diffractometer D16 at the Institut Laue Langevin, Grenoble, and on the small-angle diffractometer SAD at IPNS, Argonne National Laboratory. Measurements were made as a function of solution $\mathrm{D}_{2} \mathrm{O} / \mathrm{H}_{2} \mathrm{O}$ content, and for gels containing no template, $\mathrm{h}_{28}$-TPA template, and $\mathrm{d}_{28}$-TPA template. The concentration ratios were $1.0: 4.9: 47.4: 1850$ for $\mathrm{Al}: \mathrm{TPA}: \mathrm{Si}: \mathrm{H}_{2} \mathrm{O}$ in all the original gels except where the template was omitted. Scattered intensity profiles for some of these gels are shown in Figure 1. The contrast variation behavior is conveniently displayed using the extrapolated scattering at zero momentum transfer, $\mathrm{I}(0)$, which is proportional to $\left(\rho_{\mathrm{m}}-\rho_{\mathrm{s}}\right)^{2}$ as shown in equation 1. The contrast variation plots for d-TPA, h-TPA, and no-TPA gels are shown in Figure 2, and the measured scattering length densities are summarized in Table II. Not only is the contrast variation from such silicate solution gels sensitive to the presence of the template and the isotopic labelling of the TPA, but the scattering length density determined for the d-TPA gel is very close to that calculated for the fully crystallized d-TPA-ZSM-5 zeolite. We emphasize that at this early stage of the synthesis, the gel particles are demonstrably noncrystalline in both high-angle X-ray diffraction and FTIR spectra. On length scales of 30 - 140 A, the scattered neutron intensity shows a power-law dependence on momentum transfer of $\mathbf{- 2 . 9}$, a value which has been associated with mass-fractal scattering from densely cross-linked polymeric molecules in the small-angle scattering (of $\mathrm{X}$-rays) from silicate polymers 7 . The structure of crystalline ZSM-5 zeolite itself can be described as an extremely highly crosslinked condensation polymer in which each channel intersection accommodates a TPA ion. In the absence of the TPA, the scattering length density of the gel corresponds to that of gels formed from colloidal silica. On length scales from $140-1000 \AA$, both gels (with and without TPA) show scattering typical of fractal aggregates, with power law exponent of $-\mathbf{2 . 0}$. The onset of crystallization in the templated gel is first evident in the high-angle X-ray powder diffraction after 
it had been heated for $12 \mathrm{~h}$ at $170 \mathrm{C}$. However, it is a precursor phase other than ZSM-5 which appears first. The ZSM-5 phase is evident in the gel heated for $24 \mathrm{~h}$. A gel ficated for $18 \mathrm{~h}$ shows scattering typical of 3-dimensional particles with smooth surfaces on length scales of $60-250 \AA$, and also exhibits a scattering length density lower than that of the unheated gel. We associate this scattering with the presence of very small crystallites of the metastable precursor phase.

It is clear that the TPA molecules are incorporated into the hydrogel particles promptly upon forming a gel from soluble silicate reagent, and so for these systems the role of the template may be to catalyze the formation of a densely cross-linked aluminosilicate polymer. The TPA appears to impose a local structure in the random gel which is "embryonic" of the zeolite structure. This is consistent with the solid hydrogel reconstruction mechanism of crystallization.

SANS pattems were also measured from unheated gels formed from colloidal silica (Ludox) as the reagent, as a function of solution $\mathrm{D}_{2} \mathrm{O} / \mathrm{H}_{2} \mathrm{O}$ content, and for no template, $\mathrm{h}-\mathrm{TPA}$ template, and d-TPA template. These gels contained no added aluminium, and the TPA : $\mathrm{Si}: \mathrm{H}_{2} \mathrm{O}$ concentration ratio was $1: 25: 345$ in the templated gels. The Ludox particles themselves are smooth, dense spheres of average diameter $\geq 120 \AA$. Transmission electron micrographs show large aggregated particles in the gels. In contrast to the situation prevailing for the gels formed from sodium silicate, the SANS data on the gels formed from colloidal silica show no evidence of TPA incorporation in particles of the unheated gel which had been aged for up to two weeks at room temperature. The siattering length density of the particles in the gel containing d-TPA does not correspond to that of the crystallized zeolite, and the scattered intensity profiles for templated and untemplated gels are quite similar (Figure 3). Contrast variation plots are shown in Figure 4 for these gels containing d-TPA template, h-TPA template, and no template. The measured scattering length densities are listed in Table II, and the values are all the same within the experimental error. The power law behavior in the templated gel is consistent with scattering 
from 3-dimensional partisles with smooth surfaces. These results are consistent with a liquid phase ion transport mechanism of crystallization in these systems, or else with the requirement of an extremely long induction period for a sufficiently high concentration of the "embryonic" structures (vide supra) to develop that they might be observable in our SANS measurements.

These experiments show that small-angle neutron scattering can be a useful tool in the study of crystallization of zeolites or other molecular sieve materials from gels. Even more information can be extracted than is presented in this brief report. In principle, the technique permits : (a) measuring the sizes of solid particles in the gel in the size range $10-500 \mathrm{~A}$, corresponding to the earliest stages of crystallite nucleation and growth; (b) selective observation of one type of particle in a mixture in the solid phase of the heterogeneous gel material; (c) direct measurements on the gel in the presence of both solid and fluid phases, thus avoiding changes which might occur upon washing and drying the gel; (d) a means of investigating the structure of small particles; and (e) a method of characterizing random (amorphous) structures. More detailed results of these studies will be presented in subsequent publications.

Acknowledgements: This work is supported in part by the U.S. Department of Energy, BES-Materials Science, under Contract No. W-31-109-ENG-38. We thank the Institut Laue Langevin for the use of the neutron beam facilities, and gratefully acknowledge the assistance of Lellis Braganza and Adrian Rennie during these experiments. We thank E. I. DuPont de Nemours \& Co. for a gift of Ludox colloidal silica sol, and the PQ Corporation for a gift of sodium silicate solution. This work has benefitted from the use of the Intense Pulsed Neutron Source at Argonne National Laboratory, which is funded by the U.S. Department of Energy, BES-Materials Science, under Contract W-31-109-ENG-38. 


\section{REFERENCES}

1. Gabelica, Z,; Nagy, J. B.; Debras, G.; Derouane, E. G. In Procecdings of the Sixth Intemational Zeolite Conference; Olson, D.; Bisio, A., Eds.; Butterworths: Guilford, 1984; p. 914.

2. Gabelica, Z.; Derouane, E. G.; Blom, N. ACS Symp. Ser. 1984, 248, 219.

3. Scholle, K. F. M. G. J.; Veeman, W. S.; Frenken, P.; van der Velden, G. P. M. Appl. Catal. 1985, 17, 233.

4. Dutta, P. K.; Shieh, D. C. J. Phys. Chem. 1986, 90, 2331.

5. Dutta, P. K.; Puri, M. J. Phys. Chem. 1987, 91, 4329.

6. Jacrot, B. Repts. Prog. Phys. 1976, 39, 911.

7. Schaefer, D. W.; Keefer, K. D. In Fractals in Physics; Pietronero, L.; Tosatti, E., Eds.; North Holland: Amsterdam, 1986; p. 39.

8. Flanigen, E. M.; Bennett, J. M.; Grose, R. W.; Cohen, J. P.; Patton, R. L.; Kirchner, R. M.;Smith. J. V. Nature 1978, 271, 512. 


\section{TABLE I}

Calculated Scattering Length Densities for Zeolite Materials and Silica

Formula Fhysical Total Atomic Scattering Length Scattering Length Material Weight Density Scattering Length Density Density Equivalent (g/cc) $\Sigma b_{i}\left(10^{-12} \mathrm{~cm}\right) \quad \bar{p}\left(10^{10} \mathrm{~cm}^{2}\right) \quad D_{2} \mathrm{O}$ in $\mathrm{D}_{2} \mathrm{O} / \mathrm{H}_{2} \mathrm{O}$ (Mole frac.) (Wt.\%)
d28-TPA-ZSM-5 $6^{6}$
$\left[\mathrm{CC}_{3} \mathrm{D}_{7}\right)_{4} \mathrm{NOD}_{4}$.
266.7
4.87
$0.787 \quad 80.4$
$\left.\left(\mathrm{SiO}_{2}\right)_{96}\right)$

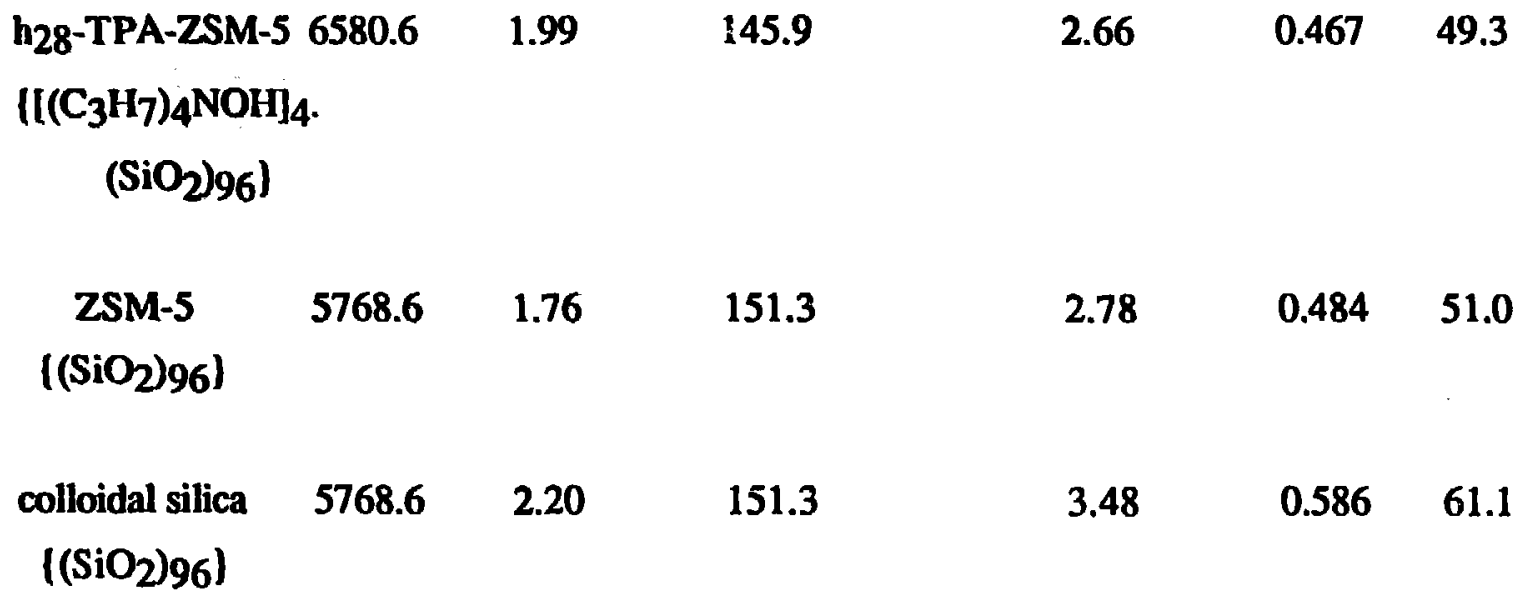

$\begin{array}{rrrrrrr}\mathrm{H}_{2} \mathrm{O} & 18.0 & 1.0 & -0.168 & -0.562 & 0 & 0 \\ \mathrm{D}_{2} \mathrm{O} & 20.0 & 1.1 & 1.915 & 6.34 & 100 & 100\end{array}$




\section{TABLE II}

\section{Measured Scattering Length Densities in Gels}

Equivalent Scattering Length Density (Wt.\% $\left.\mathrm{D}_{2} \mathrm{O} / \mathrm{H}_{2} \mathrm{O}\right)$

Gel

Sodium Silicate Gels

Colloidal Silica Gels

d28-TPA-ZSM-5

$77( \pm 1)$

$63( \pm 1)$

h28-TPA-ZSM-5

$56( \pm 1)$

$64( \pm 1)$

no-TPA-ZSM-5

$66( \pm 1)$

$64( \pm 1)$

Silica sol

$60( \pm 1)$

The stated errors are estimated from the scatter in a number of experiments. 


\section{Figure Captions}

1. SANS intensity comparisons for soluble silicate-based gels :
(a) d-TPA gel in $62 \%$ and $77 \% \mathrm{D}_{2} \mathrm{O} / \mathrm{H}_{2} \mathrm{O}$; (b) $\mathrm{h}$-TPA vs. d-TPA gels in $77 \% \mathrm{D}_{2} \mathrm{O} / \mathrm{H}_{2} \mathrm{O}$;
(c) no TPA vs. d-TPA gels in $77 \% \mathrm{D}_{2} \mathrm{O} / \mathrm{H}_{2} \mathrm{O}$.

The d-TPA gel is contrast matched in $77 \% \mathrm{D}_{2} \mathrm{O} / \mathrm{H}_{2} \mathrm{O}$.

2. Contrast variation plots of the SANS intensities for unheated soluble silicate-based synthesis gels with h-TPA template, d-TPA template, and no template.

3. SANS intensity comparisons for Ludox-based gels in $77 \% \mathrm{D}_{2} \mathrm{O} / \mathrm{H}_{2} \mathrm{O}$ :
(a) h-TPA vs. d-TPA;
(b) no TPA vs. d-TPA.

4. Contrast variation plots of the SANS intensities for unheated colloidal silica-based synthesis gels with h-TPA template, d-TPA template, and no template. 

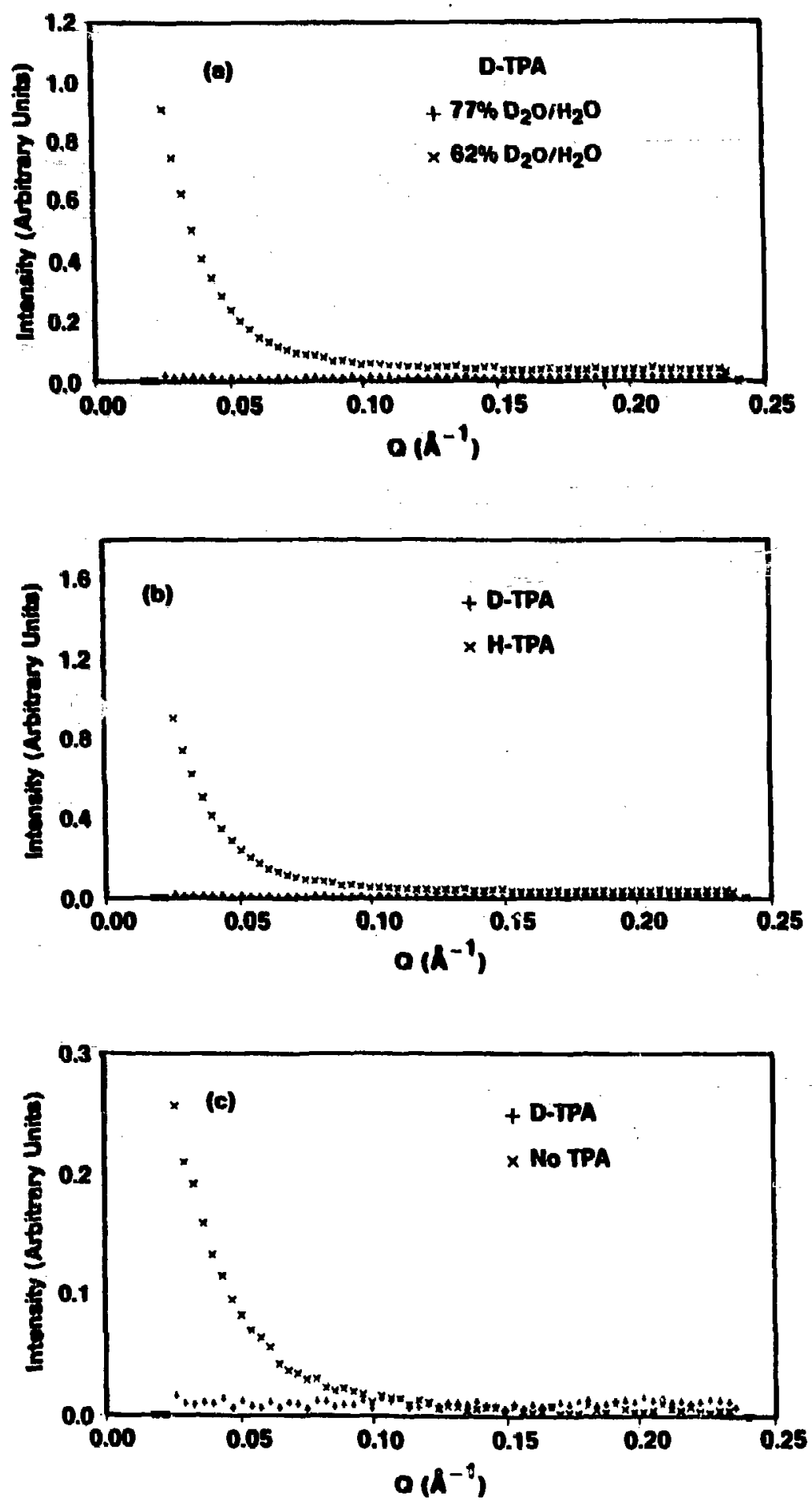

SANS (D16 at IU.) intenaity comparisons for sillicatebased gela: (a) D-TPA get in $77 \%$ and $62 \% \mathrm{D}_{2} \mathrm{O} / \mathrm{H}_{2} \mathrm{O}$, (b) D-TPA ve. H-TPA gols in $77 \% \mathrm{D}_{2} \mathrm{O} / \mathrm{H}_{2} \mathrm{O}$, and (c) D-TPA vs. no TPA gets in 77\% $\mathrm{D}_{2} \mathrm{O} / \mathrm{H}_{2} \mathrm{O}$. The D-TPA gel is conirast matched in $77 \% \mathrm{D}_{2} \mathrm{O}_{2} \mathrm{O}$. 
Contrast Variation Plots for Soluble Silicate Gels

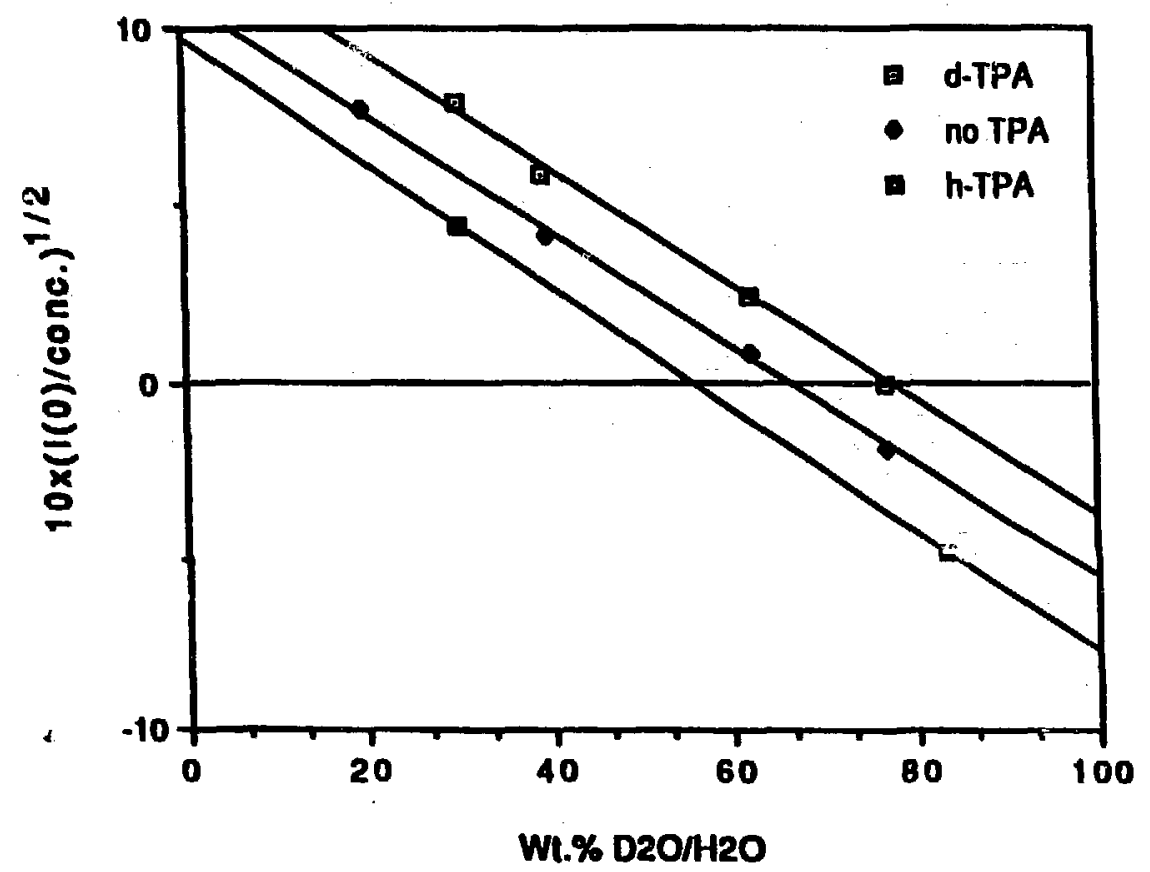

a

(-

5

$\infty$

in

$\frac{-}{6}$

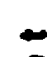

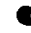

E

0 

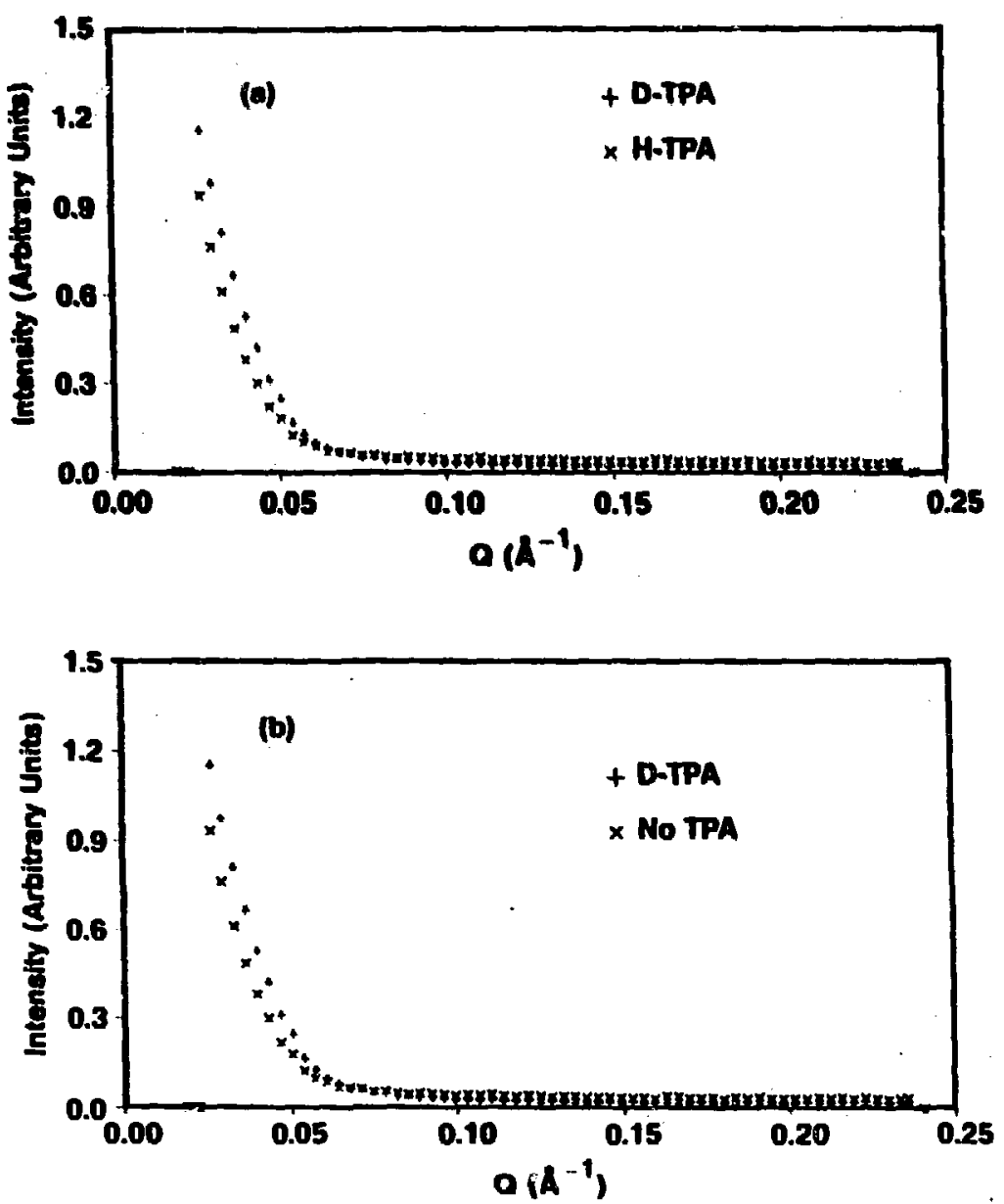

SANS (D16 at ILL) intensity comparisons for Ludoxbased gele in 77\% $\mathrm{D}_{2} \mathrm{O} / \mathrm{H}_{2} \mathrm{O}$ : (a) D-TPA va. H-TPA, and (b) D-TPA ve. no TPA. 
Contrast Variation Plots for Colloldal Silica Gels

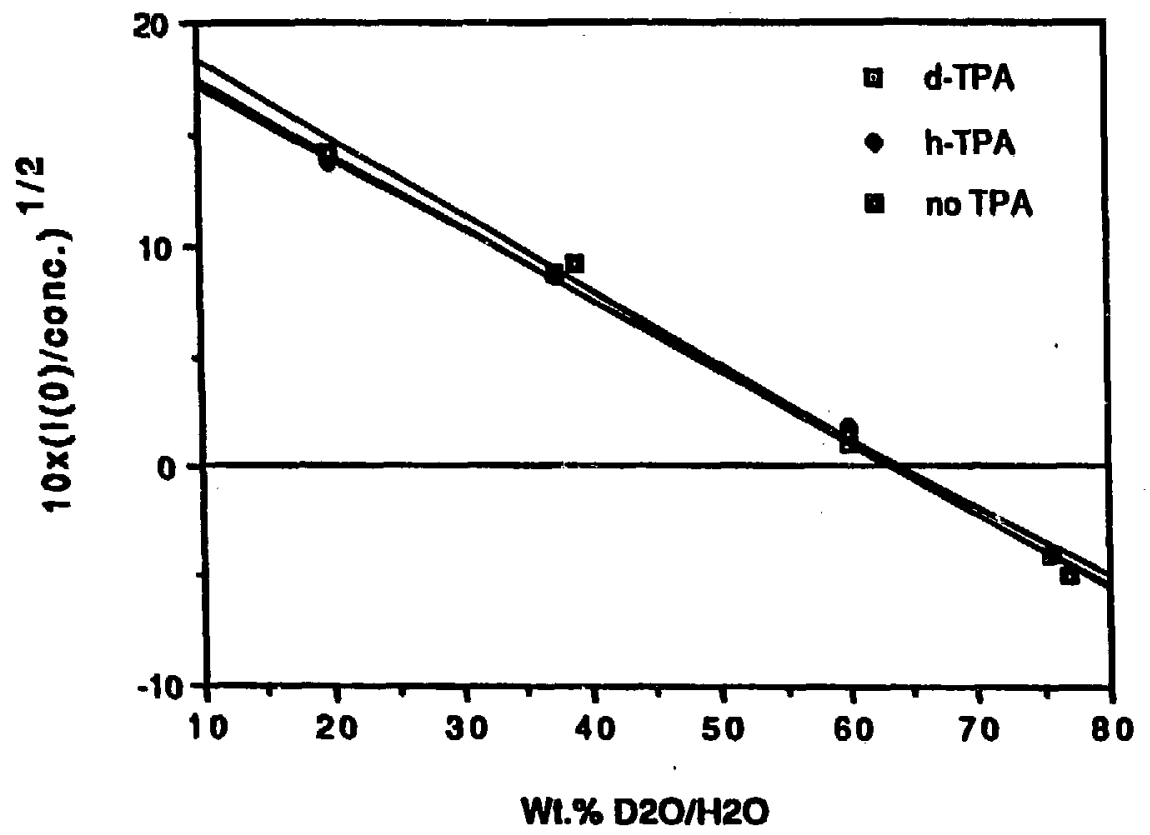

